\title{
Construcción y evaluación de un secador solar indirecto para el deshidratado de tomate saladette.
}

\author{
C. Mezquitillo-Bocanegra,B. Ruiz-Camacho, O. Martinez-Alvarez ${ }^{*}$. \\ Ingeniería en Energía, Universidad Politécnica de Guanajuato, Av. Universidad Norte s/n, Juan Alonso Cortazar Guanajuato, C.P. \\ 38483, México. \\ *omartinez@upgto.edu.mx Tel: +52-(461)4414300.Ext.4317.
}

Recibido 23 Agosto 2013, Aceptado 03 Octubre 2013

\section{Resumen}

El deshidratado de productos agrícolas es una técnica efectiva, que permite conservar y preservarlos durante tiempos prolongados, facilitando su transportación y almacenamiento. Sin embargo este proceso requiere una cantidad elevada de energía,el utilizar energías provenientes de fuentes no renovables como lo son carbón y petróleo, implica grandes problemas de contaminación. Debido a estos inconvenientes surge la necesidad de minimizar el uso de este tipo de fuentes energéticas e implementar las provenientes de los recursos naturales disponibles, la energía solar es una fuente efectiva para llevar a cabo el proceso de deshidratado de frutas, verduras, hortalizas y granos, las ventajas que ofrece este recurso energético son: reducción de emisiones contaminantes, recurso natural limpio e inagotable y gratuito.

Con la finalidad de aportar en el tema de aprovechamiento de energía solar para el deshidratado de productos agrícolas, se realizó la construcción de un secador solar indirecto tipo gabinete con reflectores, evaluando su desempeño en el deshidratado de tomate saladette. Se establecieron temperaturas para el secado de $45^{\circ} \mathrm{C}$ y $55^{\circ} \mathrm{C}$ sin y con reflectores respectivamente. Los reflectores permiten homogenizar las temperaturas en la cámara de secado, esto se ve reflejado en una mayor y uniformeremoción de humedad en las rejillas en un tiempo menor.

Palabras clave: Energía solar, secador solar indirecto, tomate saladette.

\section{Introducción}

Los productos de origen vegetal y animal son parte fundamental en la vida del hombre, por sus propiedades ricas en vitaminas minerales, fibras y antioxidantes esenciales en su alimentación [1]. El tomate saladette es una hortaliza recomendable e indispensable para una buena alimentación por sus propiedades, vitaminas $\mathrm{C}, \mathrm{A}$, $\mathrm{K}$, las de los grupos $\mathrm{B}$, y minerales como fósforo, hierro, calcio, magnesio, manganeso, zinc, cobre, potasio y sodio; además contiene licopeno, compuesto responsable del color rojo, que actúa como protector de las células ante los efectos de la oxidación [2].

En la actualidad nos enfrentamos a varias limitantes, no siempre tenemos disponibles los productos en todas las regiones del país, ni en todas las épocas del año. Debido a esta necesidad, desde hace muchos años la sociedad ha ido implementando y perfeccionando técnicas para la preservación de carnes, frutas y vegetales, utilizando el sol como fuente primordial de energía, sin embargo cuando el producto es expuesto directamente a los rayos del sol presenta una serie de desventajas como; perdida por descomposición en épocas de lluvia, largos periodos en el proceso de deshidratado, como el producto está a la intemperie el viento trae consigo un sinfin de agentes contaminantes los cuales se alojan en el producto[3,4,5]. Para poder llevar a cabo los procesos de conservación de productos se emplean diferentes métodos, entre los que destaca el deshidratado, en donde la principal característica es la remoción de humedad contenida en el producto.

Diferentes tipos de dispositivos son utilizados para este fin, existen secadores solares del tipo directo, indirecto o mixto, estos dispositivos están constituidos principalmente de dos elementos el colector solar y la cámara de secado; el colector, donde la radiación calienta el fluido que circula a través de él, en este caso el aire, generalmente el colector consta de un aislante térmico para evitar pérdidas de calor, y la cámara de secado, donde el producto es deshidratado por el aire caliente que fluye en el interior, pasando por cada una de las rejillas que alojan el producto [6]. La figura 1 muestra los tipos de secadores solares; a) Directo en el cual la cámara de secado que contiene el producto al mismo tiempo cumple la función de colector captando la radiación solar, en este tipo de dispositivos la radiación es absorbida por el producto disminuyendo considerablemente los tiempos de secado, pero afectando sus propiedades nutrimentales así como el aspecto en el producto final, b) Indirecto consta de un colector unido a la cámara de secado, el colector capta la radiación solar, calentando el aire que circula a través de él y por diferencia de densidades el aire caliente comienza a ascender hacia la cámara de secado, removiendo así la cantidad de humedad del producto contenido en cada una de las rejillas colocadas en la cámara de secado, finalmente el aire es expulsado por una chimenea, c) Mixto en este tipo de dispositivos se combina tanto el tipo directo como indirecto, donde la absorción de radiación se presenta tanto en un colector solar previo a la cámara de secado como en la misma cámara de secado, permitiendo reducir así el tiempo de secado del producto [7]. 


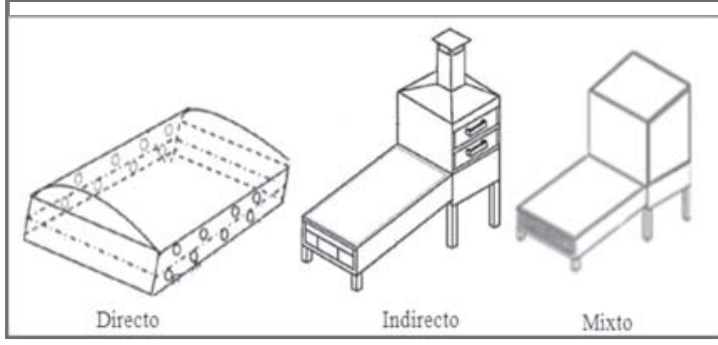

Figura 1. Tipos de secadores solares, a) Directo [8], b) Indirecto [9], y c) Mixto [10].

En este trabajo se presenta la construcción y evaluación de un secador solar indirecto tipo gabinete con y sin reflectores, para el deshidratado de tomate saladette, la gran ventaja de este tipo de secador es que la radiación solar no incide directamente sobre el tomate, permitiendo mantener el aspecto y los nutrientes en el producto deshidratado final, por otra parte los reflectores ayudan a homogenizar la temperatura en la cámara de secado y disminuir los periodos de secado.

\section{Parte experimental}

Se realizó la fabricación del secador solar indirecto tipo gabinete el cual consta de dos elementos, la cámara de secado y el colector o mueble de vidrio reforzado con madera, así como las superficies reflectoras, (ver figura 2). Se llevó a cabo la construcción del colector, el bastidor está fabricado de madera reciclada cuyas dimensiones son de $0.42 \times 0.41 \times 0.80 \mathrm{~m}$, tanto en la pared frontal como las dos laterales fue colocado vidrio, material que se emplea para que se lleve a cabo el efecto invernadero en el interior del colector, en la pared frontal se encuentra la puerta de acceso, tiene una ranura de $0.15 \mathrm{~m}$ en la parte inferior, la cual permite la entrada de flujo de aire, en la pared trasera se colocó una placa de material aislante (unicel) esto para evitar pérdidas de calor y mantener las temperaturas adquiridas en el dispositivo, en la parte superior se cuenta con una abertura que permite la salida de la chimenea de la cámara de secado de $0.09 \mathrm{~m}$ de diámetro. En seguida se llevó a cabo la construcción de la cámara de secado la cual está elaborada en su totalidad por lámina cuyas dimensiones son $0.32 \times 0.33 \times 0.725 \mathrm{~m}$, en la pared frontal se pueden apreciar 5 secciones con una separación de $0.10 \mathrm{~m}$ entre cada una de ellas, donde se albergan las rejillas, los moldes de las rejillas están construidos de madera y malla plástica lugar donde reposara el producto, sus dimensiones son $0.30 \times 0.30 \mathrm{~m}$. La cámara de secado tiene en la parte inferior de la pared trasera una ranura de $0.15 \mathrm{~m}$ de altura, esto permite la circulación de aire al interior de la cámara y a través de cada una de las rejillas, en la parte superior se encuentra la chimenea de $0.07 \mathrm{~m}$ de diámetro, medio por el cual se expulsara el aire húmedo. Los reflectores están hechos con madera y posteriormente se forraron de papel aluminio, sus dimensiones son $0.42 \times 0.70 \mathrm{~m}$, cuando el dispositivo es evaluado, los reflectores son colocados a distintos ángulos de inclinación con respecto a la superficie horizontal, de esta forma se aprovecha al máximo la cantidad de energía solar incidente en las superficies reflectoras y subsiguientemente reflejada hacia las paredes del colector, energía que es transferida y aprovechada en su totalidad en la cámara de secado, el dispositivo se coloca con una orientación hacia el sur, el reflector lateral derecho está colocado a $45^{\circ}$, el frontal a $15^{\circ}$, el lateral izquierdo colocado a $15^{\circ}$.

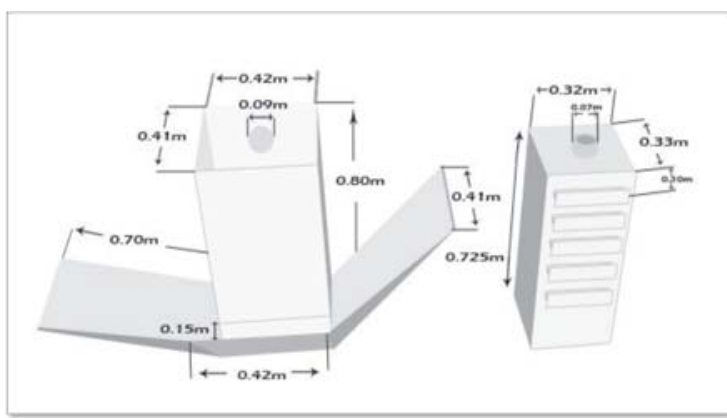

Figura 2. Secador solar indirecto tipo gabinete con y $\sin$ reflectores

La función principal del secador es remover la cantidad de humedad contenida en el tomate y de esta forma obtener un producto deshidratado, para ello se sigue un proceso cíclico el cual es descrito a continuación. El aire a temperatura ambiente ingresa por la ranura que se encuentra en la parte inferior frontal del colector, desplazándose al interior, este comienza a calentarse por el efecto invernadero producido en el interior del colector, el aire ya con una temperatura adquirida, comienza a recircular hacia la cámara de secado por la ranura inferior trasera, removiendo así la humedad contenida en el tomate, por diferencia de densidades el aire comienza a ascender por cada una de las cinco rejillas que contienen el producto, por último el aire húmedo es expulsado por la chimenea. Este proceso es repetitivo hasta que se obtiene el producto deshidratado, el hecho de colocar los reflectores nos permiten homogenizar y obtener mayores temperaturas en la cámara de secado, por consiguienteel periodo de secado fue minimizado.

El dispositivo es evaluado sin carga con y sin reflectores, con carga con reflectores, registrando parámetros de radiación solar incidente, los cuales oscilan entre $940 \mathrm{~W} / \mathrm{m}^{2}$ en su punto máximo este parámetro es muy similar cuando el dispositivo es evaluado con carga y sin carga, temperatura ambiente, las temperaturas en distintas zonas de la cámara de secado (parte superior, media e inferior) se evaluaron colocando en cada una de estas zonas un termopar tipo $\mathrm{K}$ acopladas a un multímetro KB.

Cuando el dispositivo es cargado y evaluado sin y con reflectores se seleccionaron $2 \mathrm{Kg}$ de tomate saladette, cuyas características de color y tamaño fueron muy similares. Los tomates se lavaron con agua y jabón para eliminar cualquier suciedad, estos se cortaron en rodajas, removiendo cuidadosamente las semilla, para evitar que se quedaran impregnadas en las rejillas, las rejillas fueron pesadas sin producto, se les colocaron las secciones de los tomates en las 5 rejillas y de esta manera conocer el peso inicial de producto y así poder establecer cuanto peso se perdió en cada una de las rejillas durante el 
proceso de secado. La pérdida de humedad se determina a partir de la diferencia de peso monitoreada cada hora mediante una balanza granataria, de igual manera con un colorímetro Minolta CR-400/410 se midieron los parámetros correspondientes a: luminosidad L*, color (rojo, amarillo $\mathrm{a}^{*}$ (positivo) y verde, azul b* (negativo)correspondientemente. Los datos experimentales referentes a pérdida de humedad obtenidos a través del proceso de secado son ajustados mediante diferentes modelos matemáticos: Logarítmico, Henderson y Pabis, Newton, Page, Page modificado.

\section{Resultados y discusión}

En la figura 3 se muestra la variación de temperaturas superior, media e inferior, evaluadas en el secador tipo gabinete sin carga sin reflectores, así como la variación de temperatura ambiente obtenidas durante un periodo de evaluación de nueve horas. Se observa que las temperaturas predominantes por un lapso de 5 horas en la parte superior de la cámara de secado es de $55^{\circ} \mathrm{C}$, en la parte media se tiene una temperatura de $45^{\circ} \mathrm{C}$ y en la parte inferior $35^{\circ} \mathrm{C}$, la temperatura decrece en proporción de $10^{\circ} \mathrm{C}$ en las diferentes zonas de la cámara de secado. La disminución de temperaturas se debe a que la cantidad de energía proveniente del sol se ve afectada por la distancia existente entre la parte superior e inferior del gabinete.

En la figura 4 se representan las temperaturas obtenidas en el secador con reflectores, el colocar superficies reflectoras permite aumentar significativamente las temperaturas en toda la cámara de secado, el promedio de temperatura en la parte superior es de $65^{\circ} \mathrm{C}$, en la zona media e inferior un promedio de $55^{\circ} \mathrm{C}$ por un periodo de 6 horas. Estas temperaturas son idóneas para llevar a cabo el proceso de deshidratado, minimizando considerablemente los tiempos de secado.

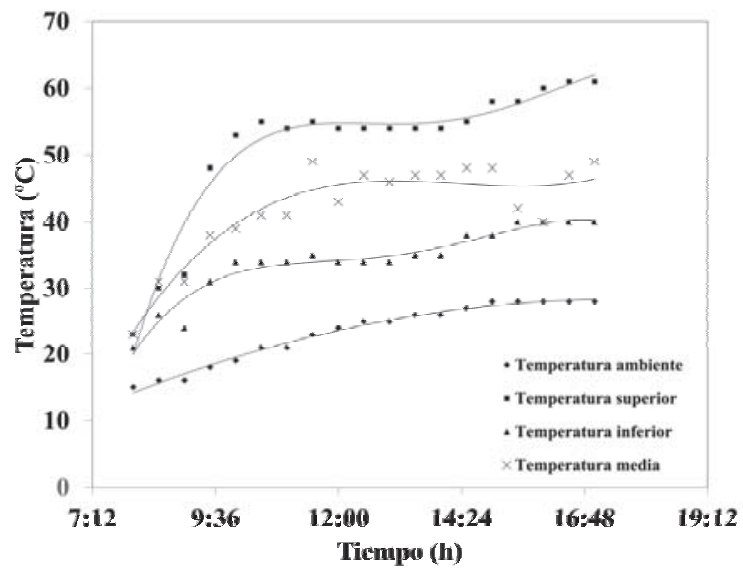

Figura 3. Variación de temperatura ambiente, superior media e inferior, evaluadas en el secador sin carga, sin reflectores.

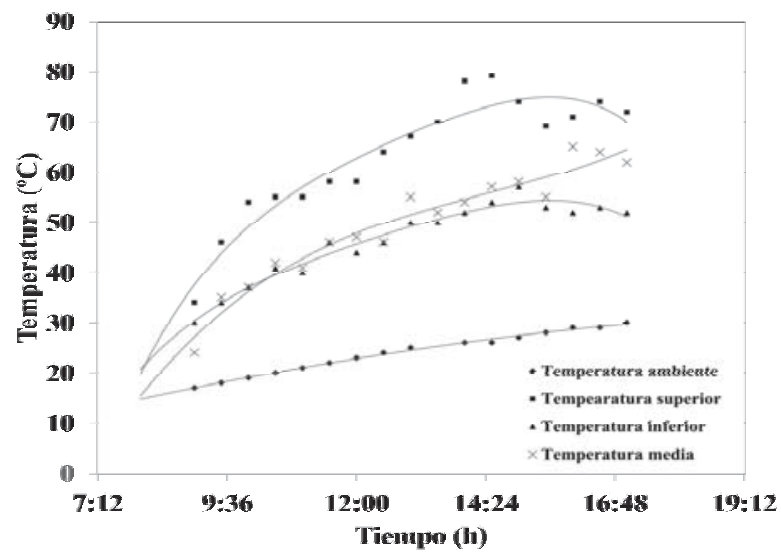

Figura 4. Evaluación de la variación de temperatura ambiente, superior, media e inferior con respecto al tiempo, sin carga con reflectores.

La figura 5 muestra las temperaturas alcanzadas cuando el dispositivo es evaluado con producto con reflectores, en la parte superior el promedio es de $55^{\circ} \mathrm{C}$ esta temperatura es similar en la parte media, en la parte inferior la temperatura por un periodo de 5 horas es de $45^{\circ} \mathrm{C}$. Estas temperaturas son óptimas para llevar a cabo la deshidratación de tomate.

La figura 6 presenta la perdida de humedad del tomate con respecto al tiempo, evaluada en el secador con reflectores. Las rejilla 1 y 5 presentan un $95 \%$ en pérdida de humedad en un periodo de casi 10 horas, el instalar los reflectores nos ayuda a que la remoción de humedad sea homogénea en las rejillas. Los resultados obtenidos son ajustados a diferentes modelos como: page modificado, newton, page, logarítmico, Henderson y pabis, de los cuales el modelo de page modificado (ecuación 1) presenta una mayor correlación $\mathrm{R}^{2} \mathrm{y} \chi^{2}$.

$$
M R=e^{-(k t)^{n}}
$$

En la tabla 1 se muestran los resultados de los datos experimentales ajustados al modelo matemático de Page modificado, así como la constante de velocidad de secado, al aplicar el modelo de page modificado.

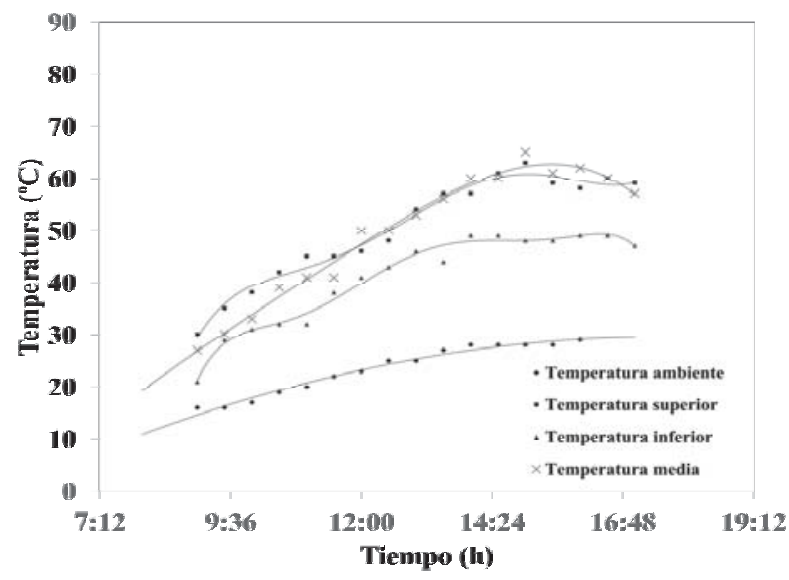

Figura 5. Variación de temperatura ambiente, superior, media e inferior con respecto al tiempo, con carga con reflectores. 
Tabla 1. Evaluación de las constantes de secado para el modelo de page modificado.

\begin{tabular}{cccccc}
\hline Modelo & Rejilla & $\mathrm{K}$ & $\mathrm{n}$ & $\mathrm{R}^{2}$ & 2 \\
\hline & 1 & 0.18136 & 1.71862 & 0.99763 & 0.00029 \\
& 2 & 0.16278 & 1.68815 & 0.99769 & 0.00025 \\
$\begin{array}{c}\text { Page } \\
\text { modificado }\end{array}$ & 3 & 0.13693 & 1.7084 & 0.99601 & 0.00034 \\
& 4 & 0.12213 & 1.77581 & 0.99481 & 0.00041 \\
& 5 & 0.17445 & 1.80066 & 0.99704 & 0.00040
\end{tabular}

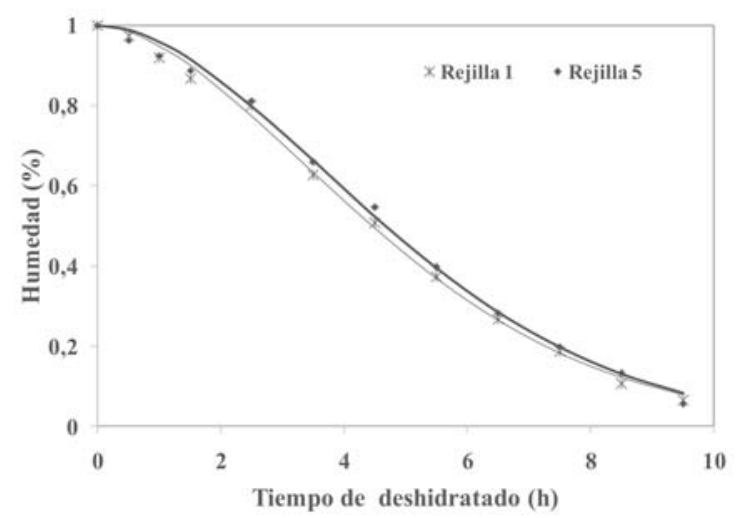

Figura 6. Perdida de humedad del tomate en la rejilla 1 y 5 con respecto al tiempo, evaluando el dispositivo con reflectores

Para determinar la calidad del tomate deshidratado uno de los parámetros importantes a considerar son el color el cual es atribuido al contenido de carotenoides que son los responsables de proporcionar el color rojo al tomate, el licopeno es el principal carotenoide presente en el producto [11]. En el proceso de secado con reflectores fueron evaluados los parámetros de luminosidad $\left(\mathrm{L}^{*}\right)$, y color $\left(\mathrm{a}^{*}, \mathrm{~b}^{*}\right)$ en cada una de las rejillas. La saturación $C^{*}$ es obtenida por la ecuación 2 la cual depende de la concentración de los colorantes o pigmentos, así mismo en la figura 7 se muestran las rodajas de tomate después del proceso de deshidratación.

$$
C^{*}=\sqrt{a^{* 2}+b^{* 2}}
$$

Las diferencias de color (E ) correspondientes a cada una de las rejillas son descritas por la ecuación 3 [12].

$$
\Delta E=\sqrt{\Delta L^{* 2}+\Delta a^{* 2}+\Delta b^{* 2}}
$$

En la tabla 2 se muestra la pérdida de peso por hora en cada una de las rejillas, evaluada de las 8:00 a las 17:00 h.
Tabla 2. Pérdida de peso (gr)

\begin{tabular}{cccccc}
\hline & & \multicolumn{5}{c}{ Rejilla } & \\
\hline Hora & 1 & 2 & 3 & 4 & 5 \\
\hline $8: 00$ & 390 & 330 & 336 & 412 & 370 \\
$9: 00$ & 386 & 326 & 362 & 410 & 366 \\
$10: 00$ & 370 & 314 & 352 & 402 & 358 \\
$11: 00$ & 360 & 304 & 344 & 396 & 350 \\
$12: 00$ & 334 & 282 & 326 & 378 & 334 \\
$13: 00$ & 316 & 268 & 314 & 366 & 322 \\
$14: 00$ & 296 & 250 & 298 & 350 & 306 \\
$15: 00$ & 280 & 234 & 282 & 336 & 294 \\
$16: 00$ & 268 & 222 & 270 & 325 & 285 \\
$17: 00$ & 256 & 212 & 256 & 312 & 278 \\
\hline & & & & & \\
\hline
\end{tabular}

La tabla 3 muestra los valores de color contenido en los tomates, cuando es deshidratado, los valores de E representan la degradación de licopeno [13], esta degradación es similar en cada una de las rejillas.

Tabla3. Evaluación L*, a*, b*, saturación y color del tomate deshidratado

\begin{tabular}{ccccccc}
\hline Tomate & Rejilla & $\mathrm{L}^{*}$ & $\mathrm{a}^{*}$ & $\mathrm{~b}^{*}$ & $\mathrm{C}^{*}$ & $\mathrm{E}$ \\
& 1 & 37.24 & 24.28 & 23.86 & 34.04 & 7.37 \\
& 2 & 41.20 & 22.88 & 24.40 & 33.44 & 10.42 \\
& & & & & & \\
Rodajas & 3 & 36.97 & 19.20 & 21.07 & 28.50 & 9.09 \\
& 4 & 46.03 & 27.15 & 32.36 & 42.24 & 7.18 \\
& & & & & & \\
& 5 & 42.59 & 21.20 & 29.36 & 36.22 & 10.36 \\
& & & & & & \\
\hline
\end{tabular}

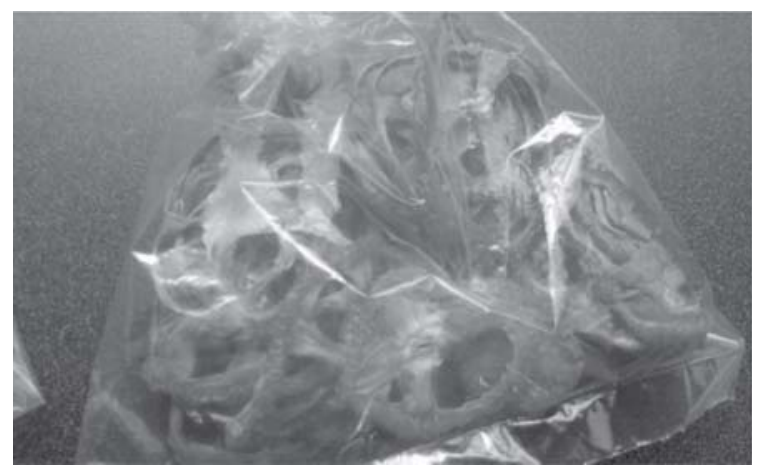

Figura 7. Apariencia de las rodajas de tomate después del proceso de deshidratado mediante un secador solar indirecto tipo gabinete con reflectores. 


\section{Conclusiones}

El costo del secador es económico, debido a que en su construcción se utilizaron materiales reciclados y de bajo costo, por lo que su adquisición y fabricación es posible por la población, lo que permite que se aprovechen al máximo los recursos naturales disponibles como lo es la energía térmica y disminuir de esta forma el uso de energías provenientes de fuentes convencionales.

Cuando el dispositivo es evaluado sin reflectores las temperaturas varían en las diferentes áreas de la cámara de secado, por tal motivo son colocados los reflectores de esta forma se minimiza este problema, homogenizando y elevando las temperaturas en la cámara de secado, lo que a su vez disminuye el periodo de secado del producto.

El modelo matemático que se ajusta los datos experimentales para predecir la pérdida de humedad contenida en el tomate cuando se evalúa sin reflectores es page modificado,

El color del tomate deshidratado no cambia considerablemente del producto sin secar, por lo que se puede considerar de buena calidad.

La energía solar es la fuente primordial para el funcionamiento de dispositivos térmicos, sin embargo esto presenta varias limitantes, como son, las condiciones climáticas, factor imprescindible e incontrolable, que afecta de forma directa al funcionamiento de los mismos, realizar dispositivos que utilicen la energía proveniente del sol cada vez es más común, debido al encarecimiento y la contaminación que provocan los combustibles fósiles.

\section{Referencias.}

1. Almanza Almanza, S, R.; Muñoz, G, F. Ingeniería de la energía solar; 2da. ed; Cromocolor: México, 2003; pp 177.

2. Candelas, C. M; Alanís, G. M; Rio, O. F; Revista mexicana de Agronegocios.2006, 10, 3 .

3. Gómez, G, M. Deshidratado de tomate saladette en un secador de charolas giratorias. Tesis de ingeniería, Universidad tecnológica de la mixteca, Oaxaca, 2009

4. Leon MA, Kumar S, Bhattacharya SC. Renew SustEnerg Rev2002, 6, 367-93.

5. Ekechukwe O, V; Norton B. Review of solar energy drying systems II: 1999, 40, 616-55.

6. Deshidratadores solares. hacia un proceso de apropiación solar de la tecnología. http://www.anesmichoacan.mx/anes2009/jueves10se pt2009/5Deshidratadores $\% 20$ Solares $\% 20 \mathrm{Hacia} \% 20 \mathrm{u}$ $\mathrm{n} \% 20$ Proceso $\% 20 \mathrm{de} \% 20$ Apropiacion $\% 20$ Solar $\% 20 \mathrm{~d}$ e\%201a\%20Tecnologia.pdf (accesado el 26 de julio de 2013).

7. Fudholi, A; Sopian K; Ruslan M.H; Alghoul M.A; Sulaiman M.Y; Renew SustEnerg Rev. 2010, 14, 130 .

8. Michael, W; Bassey; Schmidt O, G. Solar drying in Africa.International Development Research Centre, Dakar, Senegal, 1987.

9. Bolaji, BO. J Food Technol, 2005, 3, 515-600.

10. Bolaji, BO; Olalusi A, P. J Technol, 2008,11, 22531.

11. Abou E1 Hana, N. H. M.J.A.E. 2008, 25, 957-979

12. Arias, R; Lee,T.C; Logendra, L; Janes, H. J. Agric. Food Chem2000, 48, 1697-1702.

13. Olorunda, A. O; Aworh, O. C; Onuoha, C. N. J. Sci. Food Agric. 1990, 52, 447-454. 